\title{
Extreme distributed systems: from large scale to complexity
}

\author{
Alberto Montresor • Guszti Eiben • \\ Maarten van Steen
}

C The Author(s) 2012. This article is published with open access at Springerlink.com

Modern distributed systems can easily consist of hundreds of thousands of computers, ranging from high-end powerful machines to low-end resource-constrained wireless devices. We label them as "extreme distributed systems," as they push scalability and complexity well beyond traditional scenarios. The extremeness of these systems is now requiring that we reconsider our methods and techniques for their development, and, indeed, we are already witnessing a shift in thinking.

For example, Barroso and Holze [1] have made a case for a holistic design of a data center, which they essentially see as a single computer system. In their approach, the process of designing a data center is very similar to the way we have been designing processors: we need to take into account compute elements, data and control paths, storage, power sources, heating issues, and so on. As another example, groups from Lancaster University and INRIA/IRISA in Rennes are working on the integration of component-based software development with gossip-based protocols to combine structural and emergent approaches toward large-scale distributed system development [2].

It seems to be inevitable that we should concentrate more on fully decentralized solutions, as witnessed by, for example, peer-to-peer systems. Decentralized organizations often combine local decision-making with dissemination of information in order to improve the decision-making process, exemplified by many epidemic-based and other bio-inspired approaches. In this light, we are seeing much more than just ensuring that the constituents of a distributed system are properly placed, organized, and connected: the design of a distributed system is becoming fully integrated with

G. Eiben · M. van Steen $(\varangle)$

VU University, Amsterdam, The Netherlands

e-mail: steen@cs.vu.nl

A. Montresor

University of Trento, Trento, Italy 
the design of application-level algorithms. One could argue that this approach is akin to the ultimate cross-layer design in which layers consist only at an abstract logical level, but which are barely conceivable anymore at deployment time.

As a next step, we envisage that system and algorithm design will go hand-in-hand by means of a partly bottom-up approach in which computing elements are glued together into a full-fledged system that can scale beyond imagination. These new kind of systems exhibit emergent complex behavior where self-organization, optimization, and adaptation on-the-fly are inherent properties. This poses unprecedented challenges for the design and management of such systems.

For this special issue on extreme distributed systems we have solicited papers that cover this new development approach. From the many submissions, we have been able to make a selection of papers that more or less reflect what we may be expecting for the near future. The selected papers encompass topics on peer-to-peer overlay organization and management, decentralized and autonomic resource management, clustering in highly dynamic environments, privacy-preserving iterative computation in peer-to-peer networks, and incentive-based decentralized streaming. There were also many submissions suggesting more traditional approaches, yet most proposals along these lines were suffering from obvious scalability problems.

It is the mix of submissions that illustrates that we are at the verge of a new computing era, one in which we will discover new problems and need to seek for truly new solutions. The traditional boundaries between computer-systems design, software engineering, computational and artificial intelligence, heuristics, and game theory, to name but just a few, will have to be crossed and broken down if we are to succeed. The papers in this issue are good examples of how we may proceed.

Open Access This article is distributed under the terms of the Creative Commons Attribution License which permits any use, distribution, and reproduction in any medium, provided the original author(s) and the source are credited.

\section{References}

1. Barroso LA, Hölze U (2009) The Datacenter as a computer: an introduction to the design of Warehousescale machines. Morgan and Claypool, San Rafael, CA

2. Lin S, Taiani F, Bertier M, Blair GS, Kermarrec A-M (2011) Transparent componentisation: highlevel (re)configurable programming for evolving distributed systems. In: Proceedings of the 2011 ACM symposium on applied computing, ACM, New York, pp 203-208 